\title{
Objective detection of hemifield and quadrantic field defects by visual evoked cortical potentials
}

\author{
Michael S Bradnam, Donald M I Montgomery, Aled L Evans, David Keating, \\ Elizabeth A McClure, Bertil E Damato, Robert McFadzean
}

\begin{abstract}
Aims/Background-An objective method for detecting hemifield and quadrantic visual field defects has been developed using steady state visual evoked cortical potentials (VECPs), an adaptive noise canceller (ANC), and Hotelling's $t^{2}$ statistic. The purpose of this study was to determine the sensitivity and specificity of the technique.
\end{abstract}

Methods-Nine subjects (mean age 44 years) were investigated with field loss due to a variety of causes including both anterior and posterior visual pathway lesions. Dynamic perimetry was performed by means of a Goldmann or Tübingen perimeter. VECP recordings were made from each visual field quadrant $\left(23^{\circ} \times 23^{\circ}\right)$ by means of a steady state reversing checkerboard $(7 \cdot 7 \mathrm{rev} / \mathrm{s})$. The central $5^{\circ}$ of the visual field and the vertical and horizontal meridians were masked during these measurements. Recordings were made from three electrode sites, positioned over the visual cortex, relative to a mid frontal electrode. Each recording lasted 2 minutes, during which time fixation was monitored. The data from each recording were divided into 4 second segments, and the amplitude and phase of the VECP signal measured using the ANC. Hotelling's $t^{2}$ statistic was applied to determine the probability of signal detection. Receiver operating characteristic curves were used to find the optimum signal detection threshold for identification of the visual field defects.

Results-The results of the study confirmed patterns of subjective visual field loss. The technique had a sensitivity and a specificity of $81 \%$ and $85 \%$, respectively, for detecting 'non-seeing' areas in the inferior visual field, and $82 \%$ and $89 \%$, respectively, for detecting 'non-seeing' areas in the superior visual field.

Conclusion-These results demonstrate that the technique is of potential clinical value to ophthalmologists and neurologists when subjective perimetry is not possible.

(Br F Ophthalmol 1996; 80: 297-303)

Neurological Sciences,

Southern General

Hospital, Glasgow

R McFadzean

Correspondence to:

Dr Michael S Bradnam,

Department of Clinical

Physics and Bio-Engineering,

Yorkhill NHS Trust,

Glasgow G3 8SJ.

Accepted for publication

30 November 1995 ment of visual evoked cortical potential (VECPs) for objective investigation of the visual field. ${ }^{1-11}$ Early studies used focal flash stimuli to perform detailed field analysis. ${ }^{1-4} 7811$ Despite some impressive results, ${ }^{78}$ the technique has
Many workers have attempted to use meas been limited by problems with stray light, the small amplitude of the signal from extramacular stimulation, and long test durations.

Pattern reversal stimuli have provided an effective alternative to flash stimuli for analysis of visual field defects. 56910 The amplitude of the full field pattern reversal VECP is larger than the flash VECP and has a smaller interindividual range. ${ }^{56912}$ Furthermore, the pattern reversal stimulus is isoluminant, so large areas of the visual field cannot be inadvertently stimulated by scattered light.

Visual field abnormalities due to chiasmal and retrochiasmal pathology can be detected by studying the scalp distribution of the VECP in response to full field and hemifield pattern reversal stimulation. ${ }^{6}$ In normal subjects, the VECP is distributed symmetrically about the midline of the occipital scalp. Visual field defects are associated with characteristic 'crossed' or 'uncrossed' asymmetries in the VECP distribution, depending on whether the pathology affects chiasmal or retrochiasmal nerve fibres. ${ }^{6}$ Blumhardt et al ${ }^{6}$ concluded that the technique provided an objective and clinically valuable means of detecting visual field defects, and it subsequently found widespread clinical use. ${ }^{12}$ This technique could not, however, be considered as a direct replacement for conventional subjective perimetry because it did not provide detailed information of the depth, shape, or position of scotoma. ${ }^{13} 14$ The clinical importance of the VECP analysis technique lay instead in the complementary information it provided. ${ }^{13}$ VECP measurements proved to be more sensitive than subjective perimetry for detecting compressive lesions ${ }^{1315}$ and were also useful for differentiating between functional and organic visual impairment. ${ }^{16}$

A few clinical studies showed that quadrant pattern reversal stimuli further improved the detection of visual field defects. ${ }^{5910}$ Cappin and Nissim ${ }^{5}$ studied 21 patients with glaucoma, and showed clear changes in the phase and amplitude of VECPs recorded from quadrants containing visual field defects. In a subsequent study of 12 patients, Howe and Mitchell ${ }^{9}$ found that quadrantic field stimulation also made a substantial improvement in the detection of homonymous visual field defects resulting from cerebrovascular accident and migraine. Yanashima ${ }^{10}$ demonstrated homonymous hemianopia and quadrantanopia in a small group of patients.

The above quadrant field studies ${ }^{5910}$ all used steady state VECPs. Steady state VECPs are obtained from rapid stimulation rates (in excess of about five per second) in which 


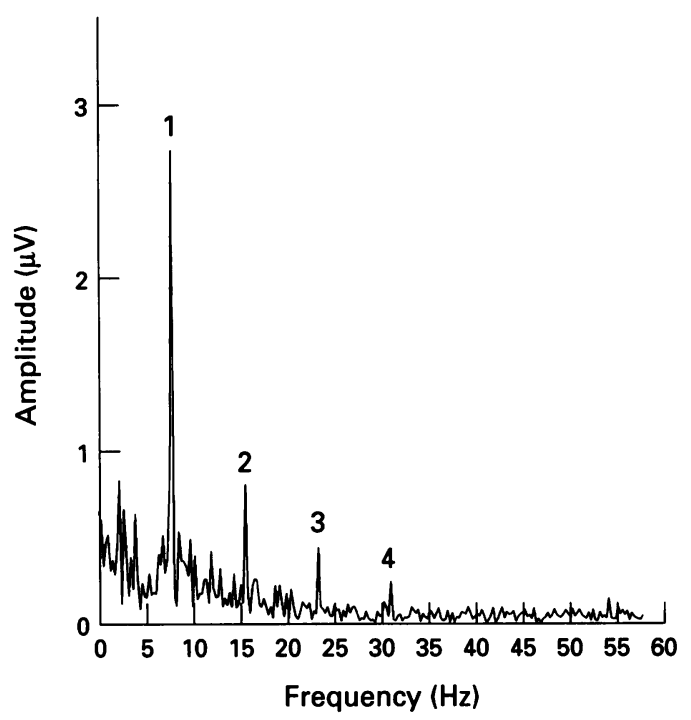

Figure 1 Example of the spectral content of a steady state visual evoked cortical potential (VECP) recording, obtained at a stimulus frequency of $7 \cdot 71$ reversals/s. This figure illustrates how the signal to noise ratio is optimised by analysis in the frequency domain. The VECP signal has discrete components at harmonics of the stimulus frequency (for example, peaks 1,2,3, and 4), while the noise from electroencephalographic and muscle activity is distributed across the whole frequency spectrum.

individual cortical responses interact and produce a periodic signal with discrete components at the stimulus frequency and its harmonics. VECPs are masked by spontaneous noise comprising electro-encephalographic (EEG) activity and muscle noise, and are usually recovered from the noise by averaging many responses. ${ }^{17}$ However, when steady state stimuli are used, the VECP signal to noise ratio (SNR) can be optimised by analysis in the frequency domain (Fig 1). ${ }^{18}{ }^{19}$ Yanashima ${ }^{10}$ took advantage of this property in his study, using the Fourier transform for signal measurement. Quadrant field studies could be further improved by using objective statistical methods to detect the VECP signal. Such a method has been developed using frequency domain analysis and Hotelling's $t^{2}$ statistic. ${ }^{20} 21$ The purpose of the current study was to determine the sensitivity and specificity of the technique for the detection of hemifield and quadrantic visual field defects in a range of subjects with pathology affecting different parts of the visual pathway.

\section{Materials and methods}

Nine subjects, two males and seven females, ranging in age from 17 to 69 years (mean 44 years) were investigated. All subjects gave informed consent for their participation in the study. The referring criteria were that the subjects had hemifield or quadrantic visual field defects and an acuity of $6 / 18$ or better. A good visual acuity was necessary to enable accurate fixation. Most subjects were referred from the neuro-ophthalmology clinic at the Institute of Neurological Sciences in Glasgow. Their pathology is detailed in Figure 2A together with their visual fields. All subjects underwent subjective dynamic perimetry by means of a Goldmann perimeter (I2e, I4e, V4e targets) or Tübingen perimeter (1.0W10, 1.0W00, 0.4W00 targets).

A specially developed system for stimulus presentation and response recording was used for the study. ${ }^{22}$ Stimuli were presented on a computer monitor screen and subjects were optically corrected for a viewing distance of 40 $\mathrm{cm}$. Monocular recordings were made from each eye, for separate stimulation of each field quadrant. A check size of 90 minutes of arc was used and the stimulus field subtended $23^{\circ} \times 23^{\circ}$ (Fig 3). A foveal mask with a radius of $5^{\circ}$ was used to prevent cortical stimulation due to central sparing of visual field defects. Masks, $2^{\circ}$ wide, were used along the vertical and horizontal meridians to prevent inadvertent stimulation of areas not under test owing to the overlap of ganglion receptive fields and slight losses of fixation. The stimulus frequency was $7 \cdot 71$ reversals per second, which is just below the EEG alpha frequency band. The pattern contrast was $99 \%$, and the mean luminance was $20 \mathrm{~cd} / \mathrm{m}^{2}$. A grey background was generated on the screen, with a luminance of $10 \mathrm{~cd} / \mathrm{m}^{2}$, equal to the mean pattern luminance. This background minimised retinal stimulation due to any scattered light. A fixation letter was normally used, but this was replaced with a spot for subjects with poor acuity. Fixation was monitored by watching eye movements and by using a simple fixation task in which subjects reported momentary changes in the colour of the fixation target. This fixation task also helped the subject to concentrate and reduced fatigue. The experiments were performed in a darkened room, with an ambient illumination of 10 lux.

In order to maximise the probability of signal detection, six recording channels were used (Fig 4). A reference electrode was positioned on the scalp within the hairline.

The data sampling frequency was $247 \mathrm{~Hz}$, one stimulus period corresponding to 32 data sample points. The recording time was 2 minutes. The amplitude and phase of the VECP signal at the stimulus reversal frequency were measured in the frequency domain by means of an adaptive noise canceller (ANC). ${ }^{2021}$ An ANC is a self optimising band pass filter. ${ }^{2324}$ The output of the ANC was vector averaged over consecutive 4 second periods. Each vector average comprised a two dimensional estimate of the sine and cosine amplitude components of the signal. The probability of signal detection was then determined by means of a two dimensional $t$ test, Hotelling's $t^{2}$ statistic. ${ }^{25} 26$ A signal was detected if the confidence interval of the mean signal amplitude from any one recording channel excluded zero.

The test sensitivity and specificity were calculated for different signal detection thresholds and used to construct receiver operating characteristic (ROC) curves. ${ }^{27}$ One curve was calculated for the inferior field quadrants and another for the superior field quadrants. These curves were in turn used to determine the optimum signal detection thresholds for identification of the quadrants containing visual field defects. Assuming that test sensitivity and specificity were equally important, then the 
optimum signal detection thresholds were indicated by the points on the curves that were closest to the top left corner of the ROC space.

\section{Results}

Figure 5 illustrates the sine and cosine amplitude values obtained from two typical recordings. Figure 5A illustrates a recording in which a signal was detected by means of Hotelling's $t^{2}$ statistic with a probability greater than $99.9 \%$, while Figure 5B illustrates a recording in which the probability of signal detection was less than $90 \%$. Figure 2B illustrates the probability of signal detection from the recording channels with the largest SNR, for all quadrants tested. The largest signal amplitudes obtained from each quadrant are shown in Figure 6 . The largest signals were usually recorded from the same channels as those with the largest SNR.

The test sensitivity and specificity were calculated for a $90 \%, 95 \%, 97.5 \%, 99 \%$, and $99.9 \%$ probability of signal detection. Figure 7 illustrates the resultant ROC curves generated for the inferior and superior field quadrants. In the inferior field quadrants (Fig 7A), the optimum sensitivity and specificity were obtained with a $99 \%$ probability of signal detection (corresponding to a sensitivity of $81 \%$ and a specificity of $85 \%$ ) while in the superior field quadrants (Fig 7B), the optimum sensitivity and specificity were obtained with a $97.5 \%$
A

Subject Pathology

1 Bilateral glaucoma

2 Pituitary chromophobe adenoma

3 Parieto-occipital haematoma

4 Parietal arteriovenous malformation
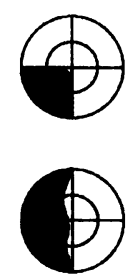

agrammatic
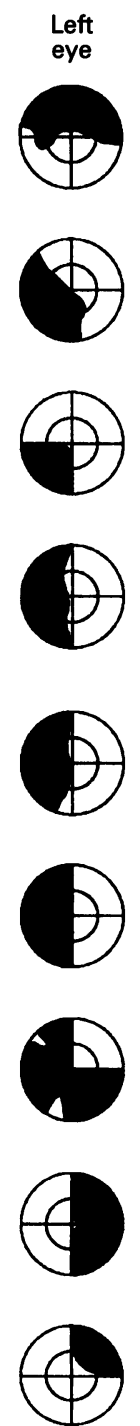

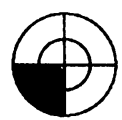
outline of visual fields
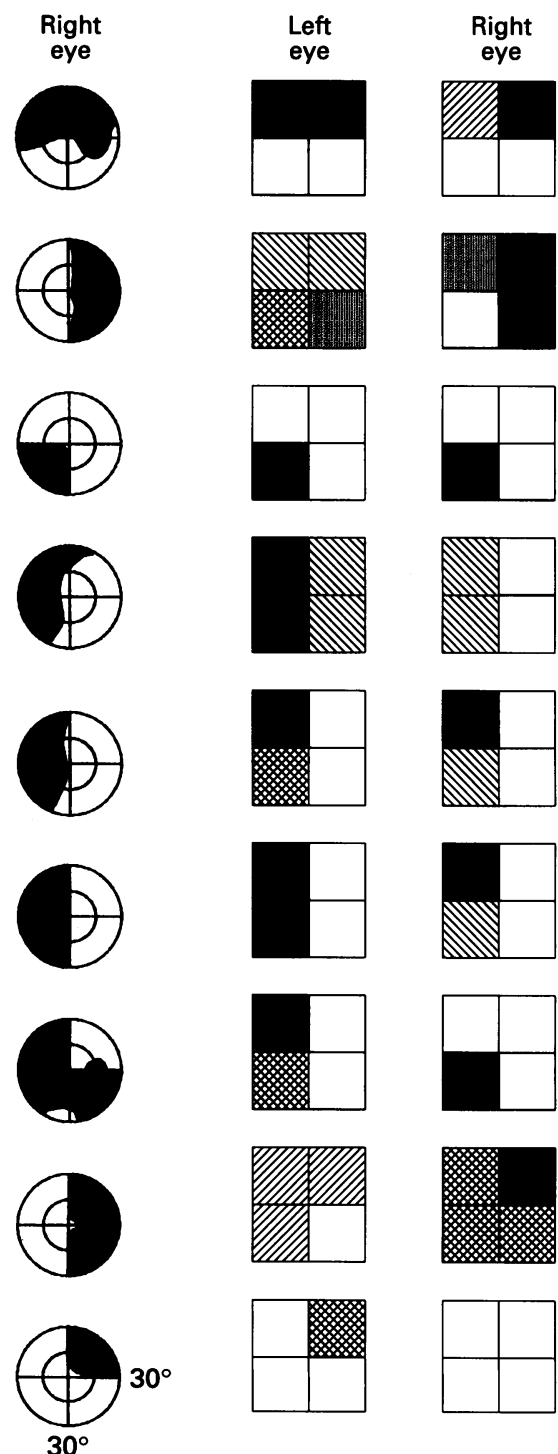

Probability of signal detection
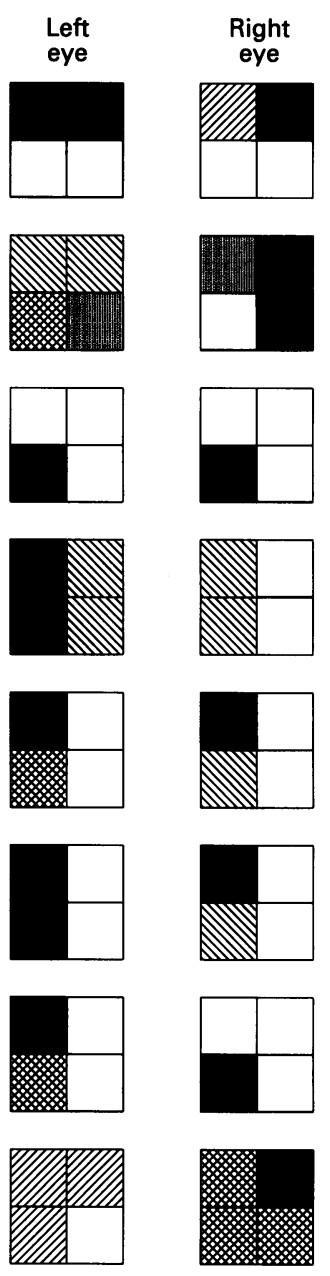

= Visual field defect

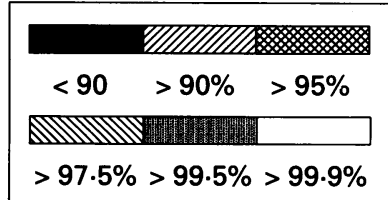

Figure 2 (A) Summary of the subjects investigated; pathology and diagrammatic representation of the central $30^{\circ}$ of the visual field and $(B)$ summary of the test results illustrating the probability of signal detection. 


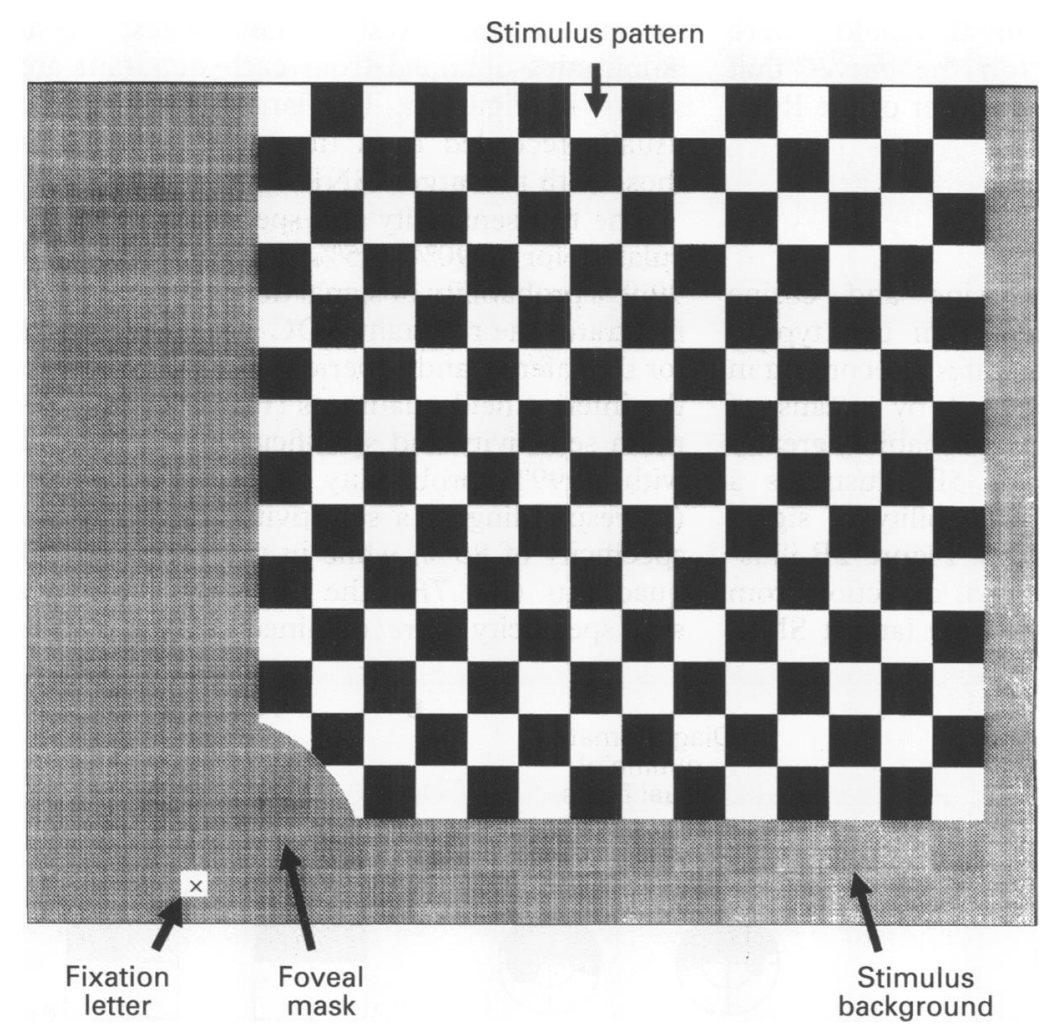

Figure 3 Quadrant field stimulus. This example illustrates the stimulus used for the right superior field quadrant.

\section{Discussion}

This study has demonstrated that the system developed in this laboratory can be used to detect hemifield and quadrantic visual field defects. The results corresponded well with the patterns of field loss mapped using conventional subjective dynamic perimetry. The main novel aspects of the system were the use of objective signal detection methods and the use of a novel technique to monitor fixation.

The optimum signal detection threshold for identifying visual field defects varied from subject to subject, and this was due to interindividual variation of the VECP SNR. The SNR was affected by variations in both the VECP amplitude and background noise. Some subjects (for example, subjects 5 and 9) had large VECP signals which were detected with a higher probability than those from other subjects (for example, subjects 2 and 8). Further variations in SNR were caused by differences in the visual field defects encountered, some visual field defects being incomplete (for example, subjects 4,7 , and 9 ). The relatively large stimulus size limited the resolution of the technique in such cases. The resolution of smaller incomplete field defects would require the use of smaller stimulus areas as described below.

Efforts were made to constantly monitor fixation, but undetected losses of fixation may have contributed to the detection of signals from 'non-seeing' areas. To avoid fixational losses an improved method for continuously checking fixation, such as video photography or electro-oculography, could be introduced.

The ROC curves provide an analysis of the relative benefits of choosing different signal

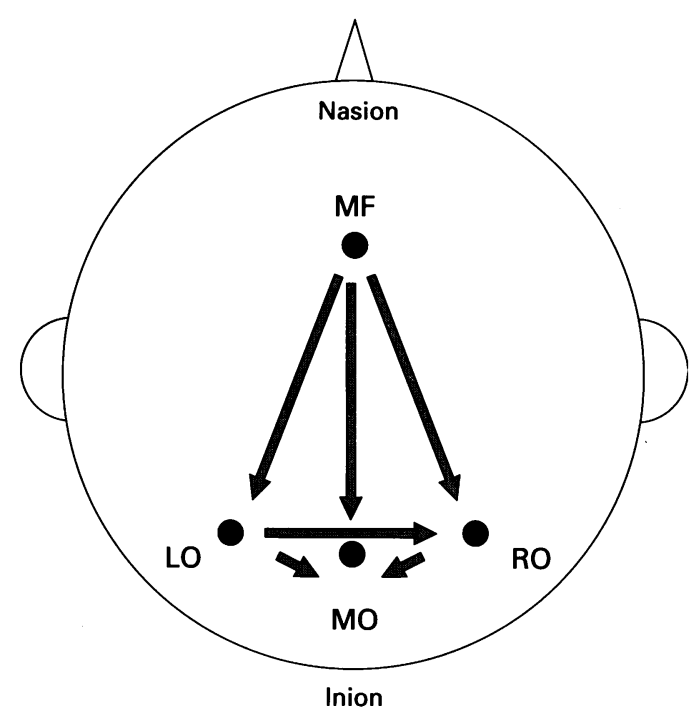

$100 \%$ larger than that recorded from the corresponding 'seeing' quadrant (subject 8) which was suggestive of poor fixation.

There was a wide interindividual variation in the cortical distribution of the VECP signal. The largest SNRs were recorded from any one of the channels (Table 1) but were most often recorded from the lateral channels, MO-RO, MO-LO, and RO-LO. The largest amplitude signals were most often recorded from the RO-LO channel (Table 2), but were never obtained from the other lateral channels MO-RO and MO-LO.
Figure 4 Electrode montage and recording channels. Three primary recording channels were used, from mid occipital (MO), right occipital (RO), and left occipital (LO) electrodes relative to a mid frontal electrode (MF). Three further recording channels were derived from these data (MO-RO, MO-LO, and RO-LO), providing a total of six channels for analysis. $M F$ was positioned $12 \mathrm{~cm}$ superior to the nasion, $M O$ was $5 \mathrm{~cm}$ superior to the inion, and $R O$ and $L O$ were $5 \mathrm{~cm}$ lateral to $M O$ (at Blumhardt et $\mathrm{al}^{6}$ ). 


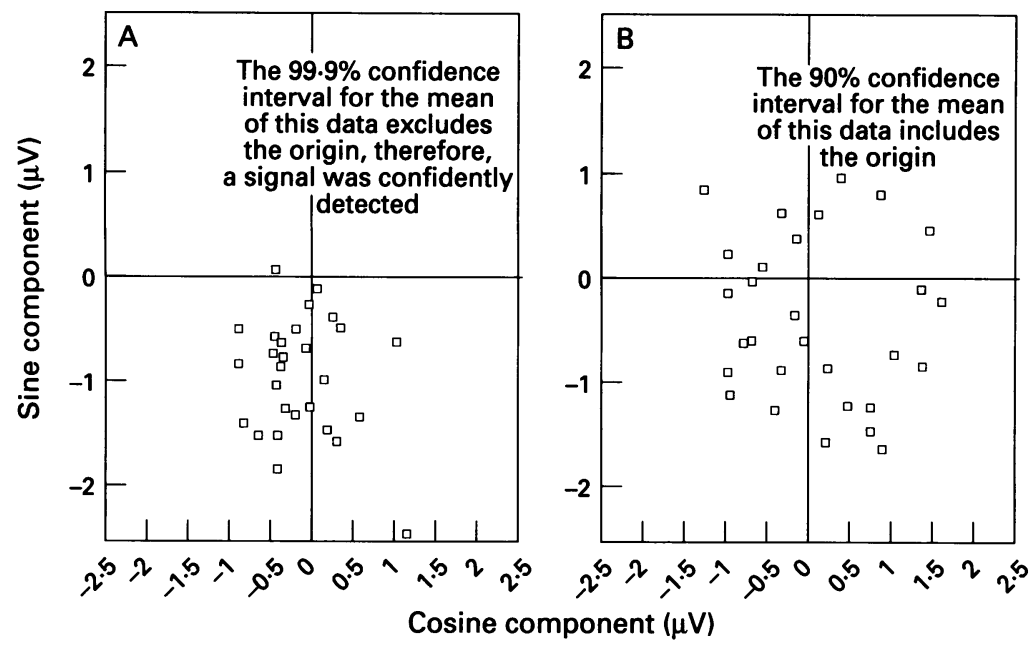

Figure 5 Example sine and cosine data from two typical recordings, each dot represents 4 seconds of data. (A) Illustrates a recording in which a signal was detected by means of Hotelling's $\mathrm{t}^{2}$ statistic with a probability greater than $99 \cdot 9 \%$ and (B) illustrates a recording in which the probability of signal detection was less than $90 \%$.

detection thresholds to identify visual field defects. In this study the optimum detection threshold was chosen assuming sensitivity and specificity were equally important. If the threshold had been set higher, the number of false positive results would have increased because the signals from subjects with low SNRs would not have been detected. On the other hand, if the threshold had been set lower, the number of false negatives would have increased because the small signals from subjects with incomplete or relative visual field defects would have been detected. Separate ROC curves were generated for the superior and inferior field quadrants because the signals from the 'seeing' quadrants in the inferior hemifield were of larger amplitude (mean $4 \cdot 2$ $\mu \mathrm{V}$, range $1 \cdot 1$ to $10 \cdot 1 \mu \mathrm{V}$ ) than those obtained from the superior hemifield (mean $2.8 \mu \mathrm{V}$, range $1 \cdot 1$ to $6 \cdot 4 \mu \mathrm{V}$ ) and were therefore easier to detect. The generated ROC curves were
Subject Visual fields

\begin{tabular}{|c|c|c|c|}
\hline & \multicolumn{2}{|c|}{$\begin{array}{l}\text { Right } \\
\text { eye }\end{array}$} \\
\hline 0.3 & 0.2 & 0.2 & 0.4 \\
\hline 4.6 & 5.6 & 3.9 & $2 \cdot 1$ \\
\hline
\end{tabular}

2

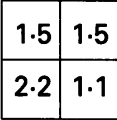

\begin{tabular}{|l|l|}
\hline 2.4 & 0.8 \\
\hline 4.3 & 1.3 \\
\hline
\end{tabular}

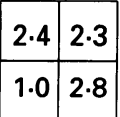

\begin{tabular}{|l|l|}
\hline 1.9 & 2.1 \\
\hline 0.8 & 3.1 \\
\hline
\end{tabular}

\begin{tabular}{|l|l|}
\hline 1.4 & 2.8 \\
\hline 1.0 & 3.4 \\
\hline
\end{tabular}

\begin{tabular}{|l|l|}
\hline 1.1 & 3.2 \\
\hline 3.4 & 4.5 \\
\hline
\end{tabular}

\begin{tabular}{|l|l|}
\hline 0.6 & 5.5 \\
\hline 0.8 & 4.4 \\
\hline
\end{tabular}

\begin{tabular}{|l|l|}
\hline 1.6 & 6.4 \\
\hline 2.1 & 4.4 \\
\hline
\end{tabular}
Subject Visual fields

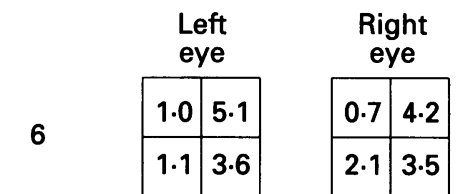

7

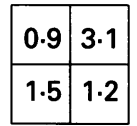

\begin{tabular}{|l|l|}
\hline 1.7 & 2.6 \\
\hline 1.1 & 1.4 \\
\hline
\end{tabular}

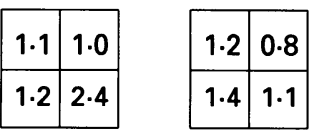

8
Figure 6 Summary of the test results illustrating the largest signal amplitude recorded from stimulation of each visual quadrant. specific to the recording variables used in the study. If, for example, the recording time was to be increased, the probability of signal detection would increase and the optimum detection threshold would also increase.

While previous clinical studies ${ }^{510}$ had used steady state stimuli, there were a number of differences in the stimuli and recording methods compared with the present study, with consequent differences in the results. Howe and Mitchell ${ }^{9}$ and Yanashima ${ }^{10}$ used smaller test fields than in the present study. Yanashima ${ }^{10}$ tested quadrants out to an eccentricity of $10^{\circ}$ and Howe and Mitchell ${ }^{9}$.tested $8.7^{\circ} \times 6.5^{\circ}$ quadrant fields. These studies used maximum check sizes of 40 minutes of arc and 50 minutes of arc, respectively, which were optimal for stimulation at a retinal eccentricity of about $5^{\circ} .^{28}$ Howe and Mitchell ${ }^{9}$ positioned the fixation target $5^{\circ}$ from the pattern. Nevertheless, owing to varying amounts of central sparing, they either reported normal or attenuated responses from the affected field quadrants. Yanashima ${ }^{10}$ presented results from two subjects, correctly demonstrating the absence of a VECP signal in the presence of a field defect. A limitation of Yanashima's stimulus ${ }^{10}$ was that it did not include a foveal mask, so it would have been relatively easy to detect signals from 'non-seeing' quadrants, owing to small losses of fixation or to central sparing. Cappin and $\mathrm{Nissim}^{5}$ stimulated larger $22^{\circ} \times 22^{\circ}$ quadrants with a 50 minute check size and a $2.5^{\circ}$ foveal mask, and found that the VECP was absent or delayed from affected field quadrants. In the present study, a 90 minute check size was used, which was optimal for stimulating more peripheral areas of the visual field, 1229 so any central sparing was therefore less likely to have affected the results.

Studies using full field and quadrant field stimuli have shown that the VECP is dominated by the response from the central visual field. ${ }^{28} 29$ For example, if a visual field quadrant of $24^{\circ} \times 24^{\circ}$ is stimulated with 90 minute checks, then approximately $60 \%$ of the VECP amplitude is due to stimulation of the central $4^{\circ}$ of the visual field. ${ }^{29}$ These same studies have shown that the optimum check size increases with retinal eccentricity. The stimulus pattern could therefore be further improved by using a 'dartboard' pattern, in which the pattern elements optimally stimulate receptive fields at each retinal eccentricity. ${ }^{30}$ The resultant improvement in SNR would enable the testing of smaller areas in the visual field and could improve the detection of incomplete hemifield and quadrantic visual field defects.

In the present study, the central $5^{\circ}$ of the visual field was masked in order to detect central sparing quadrantic visual field defects. It is obviously clinically important to test the central $5^{\circ}$ because this area is represented by just under $50 \%$ of the visual cortex. ${ }^{31} 32$ Reliable assessment of the central visual field would, however, require good fixation control combined with objective fixation monitoring.

Cappin and Nissim $^{5}$ and Howe and Mitchell $^{9}$ recorded signals from one midline 

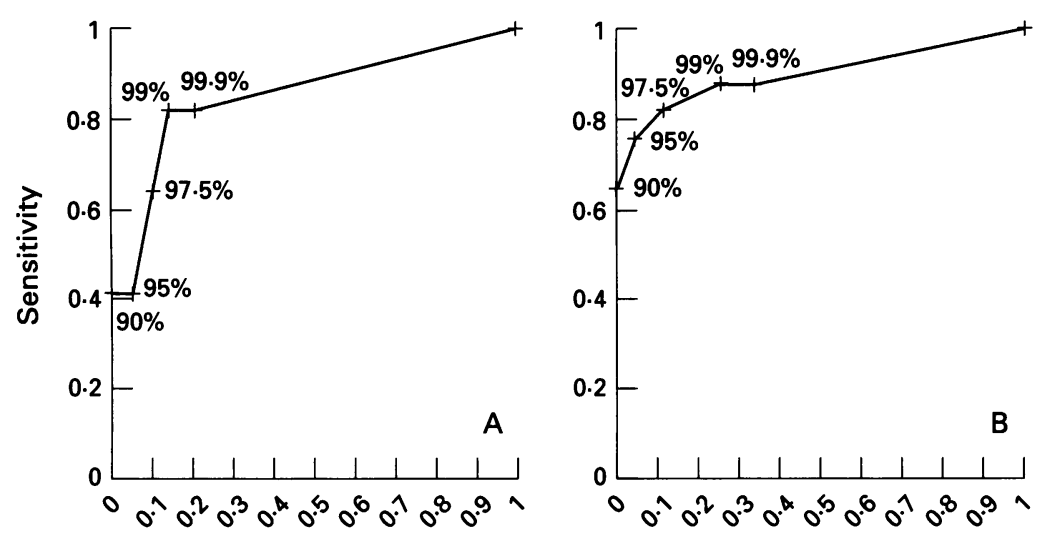

1-Specificity

Figure 7 Receiver operating characteristic (ROC) curves for $(A)$ the inferior and $(B)$ the superior field quadrants. The probability of signal detection is marked for each point on the curve.
Table 2 The number of occurrences that the maximum signal amplitude was recorded from each channel

\begin{tabular}{lc}
\hline Recording channel & Number of occurrences \\
\hline MO-MF & 5 \\
RO-MF & 6 \\
LO-MF & 3 \\
MO-RO & 0 \\
MO-LO & 0 \\
RO-LO & 25 \\
Total & 39 \\
\hline
\end{tabular}

In the present study, the VECP signal was analysed in the frequency domain to increase the SNR. ${ }^{18} 19$ The fast Fourier transform is probably the most popular tool for frequency domain analysis. However, the adaptive noise canceller has some advantages; it is a more sensitive detector, requires fewer calculations, is less computationally intensive, and requires less memory. ${ }^{21}$ The complete frequency spectrum, obtained from the Fourier transform, is not necessary for calculating Hotelling's $t^{2}$ statistic. It was found that most of the signal energy occurred at the stimulus reversal frequency, so higher harmonic components of this signal were not analysed.

The overall test time of 16 minutes used in the present study is comparable with that taken for conventional perimetry. It would, however, be possible to decrease the test time by a factor of four by stimulating simultaneously different quadrants of the visual field at different frequencies. The resultant VECP would contain components at each of the stimulus frequencies (and their harmonics), which could be analysed separately in the frequency domain. ${ }^{10181933}$

The purpose of this study was to evaluate the degree of correlation that may be obtained between conventional perimetry and perimetry using objective detection of VECPs. It was necessary, therefore, to select patients with 'barn door' field defects who were able to cooperate sufficiently to provide reliable subjective visual fields. The recording of VECPs is less demanding than conventional perimetry because the patients do not have to make decisions as to whether they can see the stimulus. Nevertheless, reliable fixation is still essential when using the current system. Clearly if this technique is to be employed in uncooperative patients a method must be devised to present the stimulus accurately irrespective of the patient's ability to fixate. To meet this objective an ophthalmoscopic delivery system is currently under development in this laboratory.

The methods described in this paper provide objective corroboration of conventional subjective perimetry findings and have the potential to provide objective information about visual fields in patients unable to cooperate adequately with subjective perimetry.

This paper is based on a presentation at the Congress of the International Neuro-Ophthalmological Society, held in Freiburg, Germany, June 1994.

Dr Bradnam was supported by the Disability and Continuing Healthcare Research Committee, Chief Scientist Office, Scottish Office Home and Health Department (grant number K/RED/E/4/1), and the Wellcome Trust (Vision Research $\mathrm{K} / \mathrm{RED} / \mathrm{E} / 4 / 1$ ), and the Wellcome Trust (Vision Research Fellowship, grant number 039072). Mr Damato is now a consultant ophthalmologist at the St Paul's Eye Unit, Royal
Liverpool University Hospital. 
1 Copenhaver RM, Beinhocker GD. Evoked occipital potentials recorded from scalp electrodes in response to foca visual illumination. Invest Ophthalmol 1963; 2: 393-406.

2 Beinhocker GD, Brooks PR, Anfenger E, Copenhaver RM Electroperimetry. IEEE Trans Bio-Med Eng BME 1966; 13: 11-8.

3 Schreinemachers HP, Henkes HE. Relation between localized retinal stimuli and the visual evoked response in man Ophthalmology 1968; 155: 2-27.

4 Henkes HE, Van Lith GHM. Electroperimetry. Doc Ophthalmol Proc Series 1973; 2: 245-51.

5 Cappin JM, Nissim S. Visual evoked responses in the assessment of field defects in glaucoma. Arch Ophthalmol 1975; 93: 9-18.

6 Blumhardt LD, Barrett G, Halliday AM. The asymmetrical visual evoked potential to pattern reversal in one half field and its significance for the analysis of visual field defects. and its significance for the analysis

7 Adachi-Usami E, Misago M, Kanayama N. Electro-perimetry by means of the scotopic VECP. Doc Ophthalmol Proc Series 1978; 15: 179-87.

8 Kojima M, Zrenner E. Determination of local thresholds in the visual field by recording the scotopic visually evoked cortical potential in man. Ophthalmic Res $1980 ; 12: 1-8$.

9 Howe JW, Mitchell KW. Visual evoked potentials from quadrantic field stimulation in the investigation of homonymous field defects. In: Barber C, ed. Evoked potentials. Lancaster: MTP Press, 1980: 279-83.

10 Yanashima K. Determination of visual field defects by the visually evoked cortical potential (VECP) decoded by fast Fourier transform (FFT). Doc Ophthalmol Proc Series 1982; 31: 427-35.

11 Elberger AJ, Spydell JD. Visual field perimetry analysis using evoked potentials in normal and corpus callosum sectioned cats. Electroenceph Clin Neurophysiol 1985; 60 249-57.

12 Spehlmann R. Evoked potential primer: visual, auditory and somatosensory evoked potentials in clinical diagnosis. Boston: somatosensory evoked

13 Halliday AM. Evoked potentials in clinical testing. Edinburgh: Churchill Livingstone, 1982.

14 Galloway NR. Evoked responses and the eye [Review]. Trans Ophthalmol Soc UK 1986; 105: 273-86.

15 Halliday AM, Halliday E, Kriss A, McDonald WI, Mushin $J$. The pattern-evoked potential in compression of the anterior visual pathways. Brain 1976; 99: 357-74.

16 Halliday AM. Evoked responses in organic and functional sensory loss. In: Fessard A, Lelord G, eds. Activities evoquees et leur conditionment chez l'homme normal et en patholoquees et leur conditionment chez l'homme normal et en path

17 Cobb WA, Dawson GD. The latency and form in man of the occipital potentials evoked by bright flashes. $\mathcal{F}$ Physiol Lond 1960; 152: 108-52.

18 Regan D. Steady-state evoked potentials. f Opt Soc Am 1977; 67: 1475-89.

19 Regan D. Human brain electrophysiology: evoked potentials and evoked magnetic fields in science and medicine. New York: Elsevier, 1989.

20 Bradnam MS, Cluckie A, Damato BE, Evans AL, Keating D, Montgomery DMI. Adaptive filtering of steady-state visual evoked cortical potentials. Presented at the XXX Symposium of the International Society for Clinical Electrophysiology of Vision, May 1992, Vienna, Austria.

21 Cluckie A, Bradnam MS, Evans AL. Measurement of steady-state visual evoked cortical potentials by means of an adaptive noise canceller. Physiol Meas 1994; 15: an adaptiv.

22 Bradnam MS, Evans AL, Montgomery DMI, Keating D, Damato BE, Cluckie A, et al. A personal computer based visual evoked potential stimulus and recording system. Doc Ophthalmol 1994; 86: 81-93.

23 Widrow B, Glover JR, McCool JM, Kaunitz J, Williams CS, Hearn RH, et al. Adaptive noise cancelling: principles and applications. Proc IEEE 1975; 63: 1692-716.

24 Widrow B, Stearns SD. Adaptive signal processing. Englewood Cliffs, New Jersey: Prentice-Hall, 1985.

25 Hotelling H. The generalisation of Student's ratio. Ann Math Stat 1931; 2: 360-78.

26 Morrison DF. Multivariate statistical methods. New York: McGraw-Hill, 1976: 128-33.

27 Metz CE. Basic principles of ROC analysis. Seminars in Nuclear Medicine 1978; 8: 283-98.

28 Harter MR. Evoked cortical responses to checkerboard patterns: effect of check-size as a function of retinal eccentricity. Vision Res 1970; 10: 1365-76.

29 Bradnam M, Keating D, Montgomery D, Evans A, Damato B, Cluckie A. Steady-state visual evoked cortical potentials from stimulation of visual field quadrants: optimizing pattern variables for the size of the field to be investigated. poc Ophthalmol 1992; 79: 151-60.

30 Barber C, Galloway NR. A pattern stimulus for optimal response from the retina. Doc Ophthalmol 1976; 10: 77-86.

31 Horton JC, Hoyt WF. The representation of the visual field in human striate cortex: a revision of the classic Holmes map. Arch Ophthalmol 1991; 109: 816-24.

32 McFadzean R, Brosnahan D, Hadley D, Mutlukan E. Representation of the visual field in the occipital striate cortex. Br f Ophthalmol 1994; 78: 185-90.

33 Regan D, Milner BA. Objective perimetry by evoked potential recording: limitations. Electroenceph Clin Neurophysiol 1978; 44: 393-7. 